Proceeding Series of the Brazilian Society of Computational and Applied Mathematics

\title{
Determinação e Estudo Comparativo de Novos Modelos Matemáticos para o Cálculo do Volume Ventricular
}

\author{
José Sérgio Domingues ${ }^{1}$
}

Departamento de Matemática, IFMG, Formiga, MG

Marcela Carvalho Gonçalves ${ }^{2}$

Departamento de Matemática, IFMG, Formiga, MG

Kálita Gonçalves da Fonseca ${ }^{3}$

Departamento de Matemática, IFMG, Formiga, MG

Alex Eduardo Andrade Borges ${ }^{4}$

Departamento de Matemática, IFMG, Formiga, MG

Resumo. O foco deste trabalho é apresentar os resultados obtidos na estimação do volume ventricular esquerdo de bovinos, pelo método de Interpolação por Splines Cúbicas, e estabelecer uma confrontação destes resultados com os obtidos pelo método de Ajuste Polinomial por Quadrados Mínimos. Os resultados indicam uma concordância significativa entre os métodos numéricos aplicados na estimação do volume ventricular e também a sua superioridade em relação aos métodos médicos tradicionais.

Palavras-chave. Modelagem Matemática, Interpolação, Spline, Volume Ventricular.

\section{Introdução}

O volume ventricular esquerdo (VVE) está relacionado ao diagnóstico de várias doenças cardíacas, como hipertensão arterial, insuficiência cardíaca e Miocardiopatia dilatada [1,3].

O objetivo desse trabalho é mostrar a possibilidade de aumentar a precisão do cálculo do VVE com base em uma modelagem por métodos numéricos. Portanto, compara-se os resultados deste trabalho utilizando o método de Interpolação por Splines Cúbicas com os obtidos com o método de Ajuste Polinomial por Quadrados Mínimos apresentados por [2].

\section{Materiais e métodos}

Com os 31 pontos do contorno superior do ventrículo esquerdo encontrados por [2] determina-se as 30 subdivisões no intervalo $[-8,8 ; 5,7]$, onde se considera a Spline Cúbica

\footnotetext{
${ }^{1}$ sergio.domingues@ifmg.edu.br

${ }^{2}$ marcela.gcarvallho@gmail.com

${ }^{3}$ kalitagf@hotmail.com

${ }^{4}$ alex.borges@ifmg.edu.br
} 
$S_{3}(x)$, cujas partes são dadas por

$$
y_{k}=a_{k}\left(x-x_{k}\right)^{3}+b_{k}\left(x-x_{k}\right)^{2}+c_{k}\left(x-x_{k}\right)+d_{k},
$$

cada uma definida em $\left[x_{k-1}, x_{k}\right]$, com $k=[1, \ldots, 30]$. O passo é $h_{k}=x_{k}-x_{k-1}$ e isso permite obter $h_{1}=0,3, h_{2}=h_{3}=\ldots=h_{29}=0,5$ e $h_{30}=0,2$. Com isso, monta-se o sistema $29 \times 29$ da forma $A X=B$ considerando a Spline Cúbica natural, $g_{0}=g_{30}=0$, e

$A=h_{k}+2\left(h_{k}+h_{k+1}\right)+h_{k+1}, X=\left[g_{0} g_{1} \ldots g_{n}\right]^{t}$ e $B=6\left(\frac{y_{k+1}-y_{k}}{h_{k+1}}-\frac{y_{k}-y_{k-1}}{h_{k}}\right)$.

A resolução do sistema pelo o software Matlab fornece os valores de $g_{k}$, utilizados para obter os coeficientes $a_{k}, b_{k}, c_{k}$ e $d_{k}$ das 30 partes da Spline Cúbica procurada.

Feito isso, determina-se a curva $S_{3}(x)$ em $\mathbb{R}^{2}$, seu sólido de revolução em linguagem Maple, seu volume pela Eq. 3 considerando $[a, b]=[-8,8 ; 5,7]$, e por fim, compara-se o resultado com o encontrado em [2].

$$
V_{s}=\pi \int_{a}^{b}\left[S_{3}(x)\right]^{2} d x
$$

\section{Resultados e Discussão}

O volume obtido pela Spline foi $V_{s}=203,7 \mathrm{ml}$, contra $V_{a}=205,1 \mathrm{ml}$ obtido pelo Ajuste Polinomial em [2]. Ambos indicam boa concordância com o volume real $V_{r}=212,5 \mathrm{ml}$. Além disso, nos dois casos, os métodos numéricos utilizados apresentam resultados consideravelmente mais precisos em relação aos métodos médicos mais usados, que indicam um volume aproximado, analisado em [2], com diferença percentual de pelo menos $16,4 \%$, sendo que para os métodos numéricos a diferença mínima foi de $3,5 \%$.

\section{Agradecimentos}

Os autores agradecem ao Programa de Bolsa de IC do IFMG-Campus Formiga.

\section{Referências}

[1] J. S. Domingues, M. P. Barbosa and M. de P. Vale, Mathematical Model for Partial Ventriculectomy, IEEE 3rd Portuguese Meeting in Bioengineering (ENBENG), (2013), DOI:10.1109/ENBENG. 2013.6518422.

[2] J.S. Domingues, K.G. da Fonseca, M. C. Gonçalves, A. E. A. Borges, Modelagem matemática e a determinação de um novo método de cálculo do volume ventricular, ForScience, v.2, 12-17, (2014).

[3] A. Galrinho, L.M. Branco, R.M. Soares, F. Miranda, A. Leal, R.C. Ferreira, Volume da aurícula esquerda: um velho cálculo ecocardiográfico com uma importância prognóstica renovada. Um estudo em doentes com miocardiopatia dilatada, Rev Port Cardiol, v.28, 1049-1060, (2009). 